\title{
A bacteriological study of CSOM in Konaseema region
}

\author{
M. Ravi Kumar Raju', V.S.A.V.Ramana Rao², G. Durga Prasad ${ }^{3}$, M.A.N. Murthy ${ }^{4}$ \\ ${ }^{1}$ Dr. M. Ravi Kumar Raju, Associate professor, ${ }^{2}$ Dr V.S.A.V.Ramana Rao, Assistant Professor, ${ }^{3}$ Dr M.A.N. Murthy, \\ Professor \& HOD, ${ }^{4}$ Dr. G. Durga Prasad, Resident. All are affiliated with Department of ENT, Konaseema Institute of \\ Medical Sciences and Research Foundation, Amalapuram, 533201, East Godavari, District, A.P, India
}

Address for correspondence: Dr. M. Ravi Kumar Raju, Email: rajumrk@yahoo.com

\begin{abstract}
Background: Chronic Suppurative Otitis Media is a disease of multiple aetiology and is well known for its persistence and recurrence in spite of treatment. Changes in the microbiological profile following the advent of sophisticated synthetic antibiotics and unscrupulous usage of antibiotics increase the relevance of reappraisal of the modern day organisms in CSOM and their in vitro antibiotic sensitivity pattern. Aim: To identify bacteriological profile in our geographical area in CSOM both in TTD and AAD types, their latest sensitivity trends to antibiotics. Materials \& Methods: 100 Patients with more than 3 months history of ear discharge selected over a period of 18 months. All patients are enquired about the history then subjected to ENT examination. From the discharging ear, pus taken with two sterile swabs and sent for direct smear and aerobic culture and sensitivity. Results: Gram negative organisms are predominant than gram positive. Pseudomonas is the most common organism found followed by Staph. aureus. Sensitivity in gram negative bacteria were mainly to Ciprofloxacin, Gentamycin and Amikacin. The gram positive organisms were highly sensitive to Amoxycillin/Clavulanic acid, Amikacin, Gentamycin, Ciprofloxacin and Tetracycline. Conclusion: Bacterial profile in discharging ears keep changing, latest bacteriological profile with antibiotic sensitivities needed to be obtained from time to time so as to treat the patient more efficiently.
\end{abstract}

Key words: CSOM, Middle ear, Culture, Antibiotic Sensitivity, Csom Bacteria

\section{Introduction}

CSOM is characterized by intermittent or persistent, chronic discharge through a perforated tympanic membrane and can be associated with cholesteatoma [1]. CSOM is a common condition in developing and underdeveloped nations. WHO has estimated the Prevalence of CSOM, as highest in India along with some other countries with $>4 \%$ and urgent attention is needed to deal with this health problem [2]. CSOM found to be one of the major cause of conductive deafness [3] and it can effect their intellectual, linguistic and psychological development $[1,4]$.

Bacteria in CSOM keeps on changing in their sensitivity and easily develop resistance to commonly and randomly used antibiotics. The wide spread use of antibiotics has precipitated in the emergence of multiple resistant strains of bacteria which can produce both

Manuscript received: $9^{\text {th }}$ Sept 2015

Reviewed: $20^{\text {th }}$ Sept 2015

Author Corrected: $4^{\text {th }}$ Oct 2015

Accepted for Publication: $10^{\text {th }}$ Oct 2015 primary and post operative infections. In these days of evidence base medicine it becomes all the more imperative that we treat with such drugs which are known to have been proved effective or guided by some research studies.

\section{Aim}

The aim of this study is to find out the organisms responsible for CSOM in both Tubo-Tympanic and Attico-Antral type, by taking the ear swab for culture and sensitivity and to find out the antibiotic sensitivity pattern of bacteria found and to reduce the morbidity and mortality of the patients.

\section{Materials and Methods}

Study Details and Inclusion Criteria: The present prospective study was conducted between January 2013 to August 2014. 100 patients with CSOM within the age group of 5 yrs to 60 yrs of both sexes attending Out Patient Department, who had not received the 
antibiotics, either locally or systemically in preceding 15 days, were selected for the study. In patients with bilateral CSOM with discharge, the ear with longest history of ear discharge is included in the study.

Exclusion criteria: Patients with ear discharge less than 3 months, chronic otitis externa with intact tympanic membrane and patients who are not willing for study are excluded.

Approval from ethical committee was taken. Consent from all the patients included in the study also taken. After history taken from the patients, ENT examination conducted for all patients. Other medical history like Diabetes mellitus, Hypertension, etc were noted. Under aseptic conditions ear discharge was collected from deeper part of the ear canal with two thin sterile cotton wool swabs from clinically diagnosed cases of CSOM. One swab sent for direct smear and another for aerobic culture and sensitivity. Antimicrobial susceptibility of the bacterial isolates to the commonly used antibiotics was done by Kirby-Bauer disc diffusion method.

Statistical analysis: Proportions, averages, and chi square test were used to find the association of the organisms with discharge and antibiotic sensitivity.

\section{Results}

Table 1: Showing the distribution of patients according to age and sex

\begin{tabular}{|l|l|l|l|}
\hline Age & Male & Female & Total \\
\hline $5-15$ & 10 & 6 & 16 \\
\hline $16-25$ & 19 & 16 & 35 \\
\hline $26-35$ & 12 & 10 & 22 \\
\hline $36-45$ & 3 & 10 & 13 \\
\hline $46-55$ & 6 & 4 & 10 \\
\hline $56-60$ & 3 & 1 & 4 \\
\hline Total & $\mathbf{5 3}$ & $\mathbf{4 7}$ & $\mathbf{1 0 0}$ \\
\hline
\end{tabular}

Of the total 100 patients 53 were male patients and 47 female. Majority of the patients were of the age group 16-25 years (35\%). Among the 5-15 age group (16\%) majority of them were male and in the 36-45 age group (13\%) majority of them were female.

Majoritity of patients in this study are from poor socio-economic status (66\%). 58 patients had discharge from the right ear, 36 patients had discharge from the left ear, and 6 of patients had discharge from both ears. In our study 82 patients had central perforation, 8 patients had marginal perforation and 10 patients had attic perforation

Table 2: Showing the distribution of Gram Positive and Gram Negative organisms

\begin{tabular}{|l|l|l|l|l|l|}
\hline $\begin{array}{l}\text { Gram positive } \\
\text { organisms }\end{array}$ & $\begin{array}{l}\text { No. of } \\
\text { cases }\end{array}$ & Percentage & $\begin{array}{l}\text { Gram negative } \\
\text { organisms }\end{array}$ & $\begin{array}{l}\text { No. of } \\
\text { cases }\end{array}$ & Percentage \\
\hline S.aureus & 20 & 21.5 & P.aeruginosa & 51 & 54.9 \\
\hline $\begin{array}{l}\text { Coagulase negative } \\
\text { S.aureus }\end{array}$ & 5 & 5.4 & Proteus & 10 & 10.8 \\
\hline- & - & - & Klebsiella & 4 & 4.3 \\
\hline- & - & - & E.coli & 3 & 3.2 \\
\hline Total & $\mathbf{2 5}$ & $\mathbf{2 6 . 9}$ & Total & $\mathbf{6 8}$ & $\mathbf{7 3 . 1}$ \\
\hline
\end{tabular}

Majority of the bacteria were Gram Negative Organisms accounts for $73.1 \%$ (of positive cultures). In 7 patients the culture is sterile. In study, Pseudomonas aeruginosa was the commonest bacterial infection accounts for 54.9\% (of positive cultures), least common bacterial infection was Escherichia coli accounts for $3.2 \%$. 
Table 3: Showing type of organisms Vs type of CSOM

\begin{tabular}{|l|l|l|l|}
\hline Bacteria & Tubo-tympanic disease & Attico-antral disease & Total \\
\hline Pseudomonas & 41 & 10 & 51 \\
\hline S.aureus & 16 & 4 & 20 \\
\hline Proteus & 9 & 1 & 10 \\
\hline Coagulase negative S.aureus & 3 & 2 & 5 \\
\hline Klebsiella & 4 & & 4 \\
\hline E.coli & 2 & 1 & 3 \\
\hline No growth & 7 & & 7 \\
\hline Total & $\mathbf{8 2}$ & $\mathbf{1 8}$ & $\mathbf{1 0 0}$ \\
\hline
\end{tabular}

Majority of the infected ears among the study patients had Tubo-tympanic disease (82.0\%) and the percentage of Atticoantral disease is $18.0 \%$. Majority of tubotympanic and atticoantral disease ears infected with pseudomonas .

Pseudomonas aeruginosa has maximum sensitivity to Ciprofloxacin (92.1\%) and high sensitivity for Gentamycin (86.3\%), Cefotaxime (74.5\%), Amikacin (68.6\%) and Cefoperazone (66.7\%). High resistance was found for Ceftazidime $(47 \%)$ and Ofloxacin $(39.2 \%)$.

Staphylococcus aureus has maximum sensitivity to Amikacin (76\%), moderate resistance to Ceftazidime and Cefoperazone (40\%), Ampicillin and Erythromycin (32\%), high resistance was found for Co-Trimoxazole (52\%).

Coagulase Negative Staphylococcus aureus has $100 \%$ sensitivity to Amikacin, high sensitivity to Gentamycin (80\%). Resistance to Amoxycillin/Clavulanic acid observed $60 \%$.

Proteus showed 100\% sensitivity to Ciprofloxacin, Gentamycin and Cefotaxime, high sensitivity to Ofloxacin (90\%), Amikacin and Co-Trimoxazole (80\%). Resistance was found to Cefoperazone (40\%) and Ampicillin (30\%).

Klebsiella has high sensitivity to Ciprofloxacin, Gentamycin, Amikacin (all being 75\%), to Cefotaxime, Cefoperazone (50\%). High resistance to Ampicillin (50\%) and moderate resistance to Co-Trimoxazole and Ofloxacin (25\%) were observed.

Escherichia coli has $100 \%$ sensitivity to Amikacin, Ciprofloxacin and Gentamycin , $66.7 \%$ sensitivity to Cefotaxime and Cefoperazone. High resistance to Ampicillin and Ofloxacin $(66.7 \%)$ and moderate resistance to Co-Trimoxazole and Tetracycline $(33.3 \%)$ were seen.

\section{Discussion}

Due to changing organisms of otitis media and sensitivity of these towards antibiotics, it has become very important to find out the organism causing the disease and the suitable antibiotic to treat them. In the present study the prevalence of CSOM is highest in the age group of $16-25$ years $(35 \%)$, followed by $26-35$ years $(22 \%)$. The results of the present study are similar to the study by Kumar .S.A. Jagadish et al, in 2012, where prevalence of CSOM was higher in age range between $26-35$, followed by $16-25$ years of age group. [5].

In 1998, Gupta Vineeta et al., found that maximum patients $(33.1 \%)$ were the age group of $21-30$ years [6], which differs from the present study. According to the study by Poorey V.K., Iyer A in 2002, the most common age group was found to be first and second decades of life [7]. Among which $1-10$ years age group was more common (46\%), which is also different from present study.

The sex distribution of CSOM in the present study is $53 \%$ males and $47 \%$ females. This shows slight male predominance in CSOM patients. The results of the present study are almost similar to the study by Kumar .S.A. Jagadish et al. in 2012, in which males were $52 \%$ and females were $48 \%$ [5].

In the present study the disease was more prevalent in the poor socioeconomic group $(66 \%)$ as compared to 
the other groups in the society. This analysis is comparable with the study of Poorey V.K. et al. where prevalence in the low socio-economic group was 50\% [7] and in the study by Kumar .S.A. Jagadish et al. where prevalence of the disease was more in the low socio-economic group (69\%) [5].

In the present series of 100 patients of CSOM, we found that, Pseudomonas aeruginosa in 51 patients was the commonest organism followed by Staphylococcus aureus in 20 patients, Proteus in 10 patients, Coagulase negative staphylococcus in 5 patients, Klebsiella in 4 patients and Escherichia coli in 3 patients. No growth was found in 7 patients.

The results of the present study are almost similar to the study by Nitin V.Deosthale et al[8]., in 2012, in which Pseudomonas aeruginosa was the most common organism (43.2\%) followed by Staphylococcus aureus $(33.7 \%)$, Klebsiella $(6.4 \%)$. No growth was found in $3.6 \%$.

In the study by Patrica N. Ayson et al, Staphylococcus aureus was the most common organism $(50 \%)$ followed by Pseudomonas aeruginosa $(33 \%)$, Proteus $(9.5 \%)$. No growth was found in 5.6\% [9]. Klebsiella, Escherichia coli and Coagulase negative staphylococcus were not isolated which differ from the present study.

In another study by Gupta Vineeta et al, Staphylococcus aureus was the most common organism (30.73\%) followed by Pseudomonas aeruginosa $(27.6 \%)$, Proteus (13.54\%), Klebsiella (9.90\%), Escherichia coli (1.56\%). No sterile culture was reported [10].

In other studies like Nitin V.Deosthale et al. [8], Patrica N. Ayson et al., in 2006 [9], and Gupta Vineeta et al., in 1998 [10], Acinetobacter, Diptheroids and Streptococcus organisms were reported which were not identified in the present study.

In the present study, majority of the organisms were Gram negative $(73.1 \%)$. Among the Gram negative organisms, Pseudomonas aeruginosa was the predominant organism followed by Proteus.

In the present study, Pseudomonas aeruginosa has maximum sensitivity to Ciprofloxacin $(92.1 \%)$ and high sensitivity to Gentamycin (86.3\%), Cefotaxime (74.5\%), Amikacin (68.6\%) and Cefoperazone (66.7\%). High resistance was found for Ceftazidime (47\%) and Ofloxacin $(39.2 \%)$ in our study.
In the study by Fliss DM et al., in 1992, all (100\%) Pseudomonas aeruginosa were sensitive to Mezlocillin and Ciprofloxacin; 99\% were sensitive to Tobramycin and Ceftazidime; and $97 \%$ were sensitive to Gentamycin, and $78 \%$ to Ceftriaxone [11]. The results of the present study are almost similar to this study.

According to the study by Vijaya D., in 2000, Pseudomonas aeruginosa showed maximum sensitivity to Amikacin and Norfloxcin $(68.1 \%$ each), followed by Gentamycin (50\%) and Cephalexin (45.4\%) [12], which differ from the present study.

In the present study Staphylococcus aureus has maximum sensitivity to Amikacin $(76 \%)$, moderate resistance to Ceftozidime and Cefoperazone (40\%), Ampicillin and Erythromycin (32\%). High resistance was found for Co-Trimaxazole (52\%).

According to Vijaya D., in 2000, Staphylococcus aureus had maximum sensitivity to Amikacin (66.6\%) [12], which is comparable to present study.

Coagulase negative Staphylococcus aureus has $100 \%$ sensitivity to Amikacin, high sensitivity to Gentamycin (75\%).Resistance to Co-Trimoxazole (50\%).

Proteus showed $100 \%$ sensitivity to Ciprofloxacin, Gentamycin and Cefotaxime, high sensitivity to Ofloxacin (90\%), Amikacin and Co-Trimaxazole (80\%). Resistance was found to Cefoperazone (40\%) and Ampicillin (30\%).

According to Vijaya D., in 2000, Proteus species were maximum sensitive to Cephalexin and Cefotaxime $(100 \%)$ followed by Amikacin (80\%) and Norfloxacin $(60 \%)[12]$, which differ from present study.

Klebsiella has high sensitivity to Ciprofloxacin, Gentamycin, Amikacin (all being 75\%), to Cefotaxime, Cefoperazone $(50 \%)$. High resistance to Ampicillin $(50 \%)$ and moderate resistance to Co-Trimoxazole and Ofloxacin $(25 \%)$ were observed.

According to Vijaya D., in 2000, Klebsiella species had maximum sensitivity to Norfloxacin $(72.7 \%)$ followed by Amikacin (59\%) and Gentamycin (50\%)[12], which differ from present study.

Escherichia coli has $100 \%$ sensitivity to Amikacin, Ciprofloxacin and Gentamycin, $66.7 \%$ sensitivity to 
Cefotaxime and Cefoperazone. High resistance to Ampicillin and Ofloxacin (66.7\%) and moderate resistance to Co-Trimoxazole and Tetracycline (33.3\%) were observed.

According to Vijaya D., in 2000, Escherichia coli had $33.3 \%$ sensitivity to Amikacin, Gentamycin and Chloromycetin [12], which differs from present study.

\section{Conclusion}

Bacteriological profile in discharging ears keeps changing with time. From time to time it becomes very important that each case of CSOM should be studied bacteriologically so as to prevent administration of unwanted antibiotics. This certainly will help in achieving dry ear and preventing complications and reduce the period of treatment thus decreasing the overall morbidity to the patient.

\section{Funding: Nil}

Conflict of interest: None.

Permission of IRB: Yes

\section{References}

1. William H. Slattery III, MD. Glasscock-Shambaugh Surgery of the Ear. 2003; 5th edition;21:422-33

2. Dr Jose Acuin et al., Chronic Suppurative Otitis Media burden of illness and management options. World Health Organization Geneva Switzerland, 2004:p14.

3. Ogisi FO. Impedance screening for otitis media with effusion in Nigerian children. J Laryngol Otol. 1988 Nov;102(11):986-8.

4. Deb T, Ray D. A study of the bacteriological profile of chronic suppurative otitis media in agartala. Indian $\mathbf{J}$
Otolaryngol Head Neck Surg. 2012 Dec;64(4):326-9. doi: 10.1007/s12070-011-0323-6. Epub 2011 Nov 5.

5. Gupta A and Gupta V. A Study of Prevalence of Complication of Suppurative Otitis Media in rural area of Loni. Indian Journal of Otology, 1996; 2 (4): $177-$ 183.

6. Kumar S. A. Jagadish, Hema Bindu. Clinical and bacteriological study of CSOM by Anaerobic Culture Methods in a Teaching Hospital; Nepalese Journal of ENT Head \& Neck Surgery, 2012; 3(2): 16-18.

7. Poorey VK, Lyer A. Study of bacterial flora in csom and its clinical significance. Indian J Otolaryngol Head Neck Surg. 2002 Apr;54(2):91-5. doi: 10.1007/BF02968724.

8. Nitin V Deosthale et al., Microbiological evaluation of CSOM. Odissa Journal of Otorhinolaryngology and HNS, 2012; 6(1):18-22.

9. Parica N.Ayson et al, CSOM: Bacteriology and drug sensitivity patterns at the Quirino memorial medical center(2004-2005):A preliminary study. Philippine J of Otolaryngology-Head and Neck Surgery,2006; 21(1,2):20-23.

10. Gupta V, Gupta A, Sivarajan K. Chronic Suppurative Otitis Media: An Aerobic Microbiological study. Indian Journal of Otology, 1998; 4(2): 79-82

11. Fliss DM, Dagan R, Meidan N, Leiberman A. Aerobic bacteriology of chronic suppurative otitis media without cholesteatoma in children. Ann Otol Rhinol Laryngol. 1992 Oct;101(10):866-9.

12. Vijaya D. Aerobes, Anaerobes and Fungi in Chronic Suppurative Otitis Media. Indian Journal of Otology, 2000; 6 (3): 55-58.

\section{How to cite this article?}

M. Ravi Kumar Raju, V.S.A.V. Ramana Rao, G Durga Prasad ${ }^{3}$, M.A.N. Murthy. A bacteriological study of CSOM in Konaseema region. Int J Med Res Rev 2015;3(9):964-968. doi: 10.17511/ijmrr.2015.19.179. 